\title{
OPEN Development and validation of a new diabetes index for the risk classification of present and new-onset diabetes: multicohort study
}

\author{
Shinje Moon ${ }^{1,4}$, Ji-Yong Jang ${ }^{2,4}$, Yumin $\mathrm{Kim}^{3}$ \& Chang-Myung Oh ${ }^{3 凶}$
}

In this study, we aimed to propose a novel diabetes index for the risk classification based on machine learning techniques with a high accuracy for diabetes mellitus. Upon analyzing their demographic and biochemical data, we classified the 2013-16 Korea National Health and Nutrition Examination Survey (KNHANES), the 2017-18 KNHANES, and the Korean Genome and Epidemiology Study (KoGES), as the derivation, internal validation, and external validation sets, respectively. We constructed a new diabetes index using logistic regression (LR) and calculated the probability of diabetes in the validation sets. We used the area under the receiver operating characteristic curve (AUROC) and Cox regression analysis to measure the performance of the internal and external validation sets, respectively. We constructed a gender-specific diabetes prediction model, having a resultant AUROC of 0.93 and 0.94 for men and women, respectively. Based on this probability, we classified participants into five groups and analyzed cumulative incidence from the KoGES dataset. Group 5 demonstrated significantly worse outcomes than those in other groups. Our novel model for predicting diabetes, based on two large-scale population-based cohort studies, showed high sensitivity and selectivity. Therefore, our diabetes index can be used to classify individuals at high risk of diabetes.

Diabetes mellitus is a chronic metabolic disorder characterized by disrupted glucose homeostasis, resulting from increased insulin resistance and/or impaired insulin secretion. People with diabetes mellitus are predisposed to metabolic disorders, such as cardiovascular disease (CVD), which affects $32.2 \%$ of all people with diabetes mellitus globally. Moreover, their complications are leading causes of morbidity and mortality ${ }^{1,2}$. The prevalence and socioeconomic burden of diabetes are rapidly increasing worldwide. Approximately 1 in 11 adults have diabetes, and $90 \%$ of people with diabetes have type 2 diabetes mellitus ${ }^{2}$.

Previous large-scale studies suggest that diet and lifestyle modifications can prevent or delay the development of diabetes mellitus in high-risk individuals by Refs. ${ }^{2,3}$. The Diabetes Prevention Program conducted in the Unites States reported that lifestyle modification reduced the incidence of diabetes mellitus by $58 \%$ compared with control after a 2.8-year mean follow-up ${ }^{4}$. Toshikazu et al. also demonstrated that lifestyle modification reduced the overall relative risk of diabetes mellitus by $44.1 \%$ in Japan ${ }^{5}$. Clinical studies conducted in China $^{6}$ and India ${ }^{7}$ have reported $42 \%$ and $38 \%$ risk reductions, respectively.

Therefore, developing risk prediction models for diabetes mellitus and identifying high-risk individuals have become a challenging issue in clinical research. To explore the risk factors and formulate predictive models for diabetes development, machine learning techniques have been widely used ${ }^{8}$. These methods help researchers discover unknown significant figures and solve scientific problems from large quantities of datasets ${ }^{9,10}$. In the fields of medical science and healthcare, machine learning provides useful classification and prediction models with high accuracy ${ }^{11}$. Recently, Hang Lai et al. proposed a risk prediction model with $84.7 \%$ area under the receiver operating characteristic curve (AUROC) from 13,309 Canadian patients ${ }^{12}$. Furthermore, Maniruzzaman et al.

${ }^{1}$ Department of Endocrinology and Metabolism, Hallym University College of Medicine, Chuncheon, Republic of Korea. ${ }^{2}$ Division of Cardiology, National Health Insurance Service Ilsan Hospital, Goyang, Republic of Korea. ${ }^{3}$ Department of Biomedical Science and Engineering, Gwangju Institute of Science and Technology, Gwangju, Republic of Korea. ${ }^{4}$ These authors contributed equally: Shinje Moon and Ji-Yong Jang. ${ }^{\varpi}$ email: cmoh@ gist.ac.kr 
built a classifying model that yielded $94.25 \%$ accuracy for the prediction of diabetes mellitus from an American diabetes dataset ${ }^{13}$.

In this study, we aimed to propose a novel diabetes index based on machine learning techniques for diabetes mellitus with high accuracy from two large community-based cohort studies. We formulated a risk classification model using logistic regression to measure the probability of diabetes presence, based on non-diabetic participants' demographic information and laboratory data from the Korea National Health and Nutrition Examination Survey (KNHANES). Thereafter, we externally validated this model by predicting new-onset diabetes mellitus in a large prospective cohort study known as the Korean Genome and Epidemiology Study (KoGES).

\section{Results}

Baseline characteristics from the KNHANES. Table 1 show the general characteristics from the KNHANES. These depict the derivation and internal validation datasets, respectively, according to gender and diabetes. Subjects with diabetes were older than those without in both datasets. In the derivation dataset, diabetes prevalence was $4.9 \%$ in men and $3.8 \%$ in women. The prevalence of obesity (Body mass index, BMI $\geq 25 \mathrm{~kg} /$ $\mathrm{m}^{2}$ ) was $38 \%$ in men (38\% in normal and $38 \%$ in diabetes) and $28.1 \%$ in women $(27.3 \%$ in normal and $47.3 \%$ in diabetes). In the internal validation dataset, diabetes prevalence was $4.6 \%$ in men and $3.9 \%$ in women. The prevalence of obesity $\left(\mathrm{BMI} \geq 25 \mathrm{~kg} / \mathrm{m}^{2}\right)$ was $40.8 \%$ in men ( $40.6 \%$ in normal and $44.5 \%$ in diabetes) and $27.6 \%$ in women (26.8\% in normal and $46 \%$ in diabetes). Subjects with diabetes in both datasets exhibited lower socioeconomic status and education, higher fasting glucose levels, as well as higher incidence of glycosuria, hypertension, and dyslipidemia than did subjects without diabetes.

Feature selection and classification model by logistic regression. Based on literature review, we identified about 40 candidate risk factors (Supplementary Table 1), in 20 variables present in both KNHANES and KoGES. Table 2 displayed the selection process by means of a univariate LR in men and women, respectively. All 20 features from Model 1 were selected as candidate variables for univariate analysis in Model 2. By means of multivariate analysis (Models 2 and 3), we identified 16 and 18 variables as diabetes risk factors to be utilized as the input features for formulating the classification model in men and women, respectively. Thereafter, based on these variables, we generated a gender-specific diabetes classification model using LR. Note that the feature selection and the formulation of the prediction model were conducted using only the derivation dataset.

We used this gender-specific diabetes classification model to calculate the probabilities of diabetes in subjects from the internal validation dataset. The area under the receiver operating curve (AUROC) was 0.941 and 0.939 in men and women, respectively (Fig. 1). The area of under the precision-recall (PR) curve was 0.475 and 0.381 in men and women, respectively (Fig. 1). Moreover, we evaluated the model performance via calibration, the agreement between observed and predicted probabilities using val.prob function in the rms package. As a result, the classification model for women was a well-calibrated model, besides the model for men was not according to the Spiegelhalter Z-test and its two-tailed $p$-values (S:p for men: 0.008; S:p for women: 0.588, Supplementary Fig. 2).

External validation of the classification model. Table 3 shows baseline characteristics of the KOGES dataset. By using our gender-specific classifying model constructed from the derivation dataset, we calculated the probabilities of the presence of diabetes in subjects from the external validation dataset. These subjects were categorized into five groups according to the probabilities of the subjects in ascending order. Figure 2 shows the cumulative incidence of new-onset diabetes. Most groups had significant differences from other groups. For both men and women, group 5 yielded significantly worse outcomes than those in other groups.

\section{Discussion}

Our novel model for the risk classification of diabetes mellitus, based on two large-scale population-based cohort studies, showed high sensitivity and selectivity. Our model yielded AUROCs of 0.941 and 0.939 in men and women, respectively. The Finnish Diabetes Risk Score (FINDRISC) model is a well-known, recommended tool for diabetes mellitus prediction ${ }^{14}$. The AUROC of the FINDRISC model was 0.77 and 0.74 in the Norwegian ${ }^{15}$ and Spanish ${ }^{16}$ populations, respectively. The Framingham Diabetes Risk Scoring Model (FDRSM) by Wilson et al. ${ }^{17}$ yielded an AUROC of 0.85 and 0.78 in middle aged American and Canadian populations, respectively ${ }^{18}$. In the Asian population, Quan Zou et al. predicted new-onset diabetes using the machine learning technique from a Chinese cohort. Their model yielded an AUROC of $0.8084^{19}$. The diabetes risk score model from the KoGES by Kim et al. ${ }^{20}$ yielded AUROC of 0.71 and 0.76 in men and women, respectively. Note that the predictive performance by our model is for the presence of DM, not the new-onset DM, thereby, somewhat outperforms compared to previous models predicting the new-onset DM. We had performed the literature-review and statistical methods to select more than 15 predictors, which are the potentially appropriate model for DM that has the complex pathophysiology.

With the help of machine learning techniques, we can handle large numbers of participant features that may have positive or negative correlations with the prevalence of diabetes mellitus. To obtain input features for our model, we used data from the KNHANES, a large-scale cross-sectional study that includes approximately 10,000 participants. As a result, we were able to use the 16 and 18 variables in men and women, respectively, during the analysis (Table 3).

Among these variables, glycosuria showed the highest odds ratio (OR) in men (OR 1.35; 95\% CI 1.32-1.39). In general, glycosuria has been used as a biomarker for renal complication in diabetes ${ }^{8,21}$, not as a predictor for diabetes. Although glycosuria is a result of hyperglycemia, it also occurs with normal blood glucose levels due to renal injury. Moreover, hyperglycemic patients can also secrete normal range glucose in their urine ${ }^{22,23}$. This implies that we need to identify a new risk factor that, despite being considered negligible, may have a significant 


\begin{tabular}{|c|c|c|c|c|c|c|}
\hline & \multicolumn{3}{|l|}{ Men } & \multicolumn{3}{|l|}{ Women } \\
\hline & Normal & Diabetes & P-value & Normal & Diabetes & P-value \\
\hline \multicolumn{7}{|l|}{ 2013-16 KNHANES } \\
\hline $\mathrm{N}$ & 5751 & 300 & & 8590 & 336 & \\
\hline Age, $y$ & $49.3 \pm 0.21$ & $65.2 \pm 0.59$ & $<0.001$ & $48.6 \pm 0.17$ & $66.4 \pm 0.57$ & $<0.001$ \\
\hline Income, $\mathbf{n}$ & & & 0.019 & & & 0.342 \\
\hline Quartile 1 & $1338(23.3)$ & $77(25.7)$ & & $1980(23.1)$ & $89(26.5)$ & \\
\hline Quartile 2 & $1446(25.1)$ & $95(31.7)$ & & $2181(25.4)$ & $88(26.2)$ & \\
\hline Quartile 3 & $1465(25.5)$ & $63(21)$ & & $2211(25.7)$ & $84(25)$ & \\
\hline Quartile 4 & $1502(26.1)$ & $65(21.7)$ & & $2218(25.8)$ & $75(22.3)$ & \\
\hline Education, $\mathrm{n}$ & & & $<0.001$ & & & $<0.001$ \\
\hline Elementary & $804(14)$ & $101(33.7)$ & & $1921(22.4)$ & $201(59.8)$ & \\
\hline Middle school & $586(10.2)$ & $54(18)$ & & $826(9.6)$ & $58(17.3)$ & \\
\hline High school & $2033(35.4)$ & $81(27)$ & & $2844(33.1)$ & $57(17)$ & \\
\hline College & $2328(40.5)$ & $64(21.3)$ & & $2999(34.9)$ & $20(6)$ & \\
\hline Smoking, pack years & $13.6 \pm 0.23$ & $22.3 \pm 1.25$ & $<0.001$ & $0.6 \pm 0.04$ & $1.2 \pm 0.3$ & 0.042 \\
\hline Alcohol, g/week & $119.5 \pm 2.28$ & $79.5 \pm 8.06$ & $<0.001$ & $28.4 \pm 0.86$ & $6 \pm 1.18$ & $<0.001$ \\
\hline Sleep duration, $\mathrm{h}$ & $6.9 \pm 0.02$ & $7.1 \pm 0.09$ & 0.001 & $6.9 \pm 0.01$ & $6.4 \pm 0.09$ & $<0.001$ \\
\hline Hypertension, $\mathrm{n}$ & $1012(17.6)$ & $174(58)$ & $<0.001$ & $1364(15.9)$ & $204(60.7)$ & $<0.001$ \\
\hline Dyslipidemia, $\mathrm{n}$ & $321(5.6)$ & $87(29)$ & $<0.001$ & $723(8.4)$ & $128(38.1)$ & $<0.001$ \\
\hline Cardiovascular disease, $\mathrm{n}$ & $193(3.4)$ & $55(18.3)$ & $<0.001$ & $188(2.2)$ & $37(11)$ & $<0.001$ \\
\hline Systolic BP, mmHg & $120.2 \pm 0.2$ & $123.3 \pm 0.98$ & 0.002 & $115 \pm 0.19$ & $126.1 \pm 0.99$ & $<0.001$ \\
\hline Diastolic BP, mmHg & $77.8 \pm 0.14$ & $71.8 \pm 0.57$ & $<0.001$ & $73.3 \pm 0.1$ & $71.4 \pm 0.51$ & $<0.001$ \\
\hline Body mass index, $n$ & & & 0.999 & & & $<0.001$ \\
\hline $\operatorname{Normal}\left(<23 \mathrm{~kg} / \mathrm{m}^{2}\right)$ & $2044(35.5)$ & $107(35.7)$ & & $4421(51.5)$ & $95(28.3)$ & \\
\hline Pre-obesity $\left(23 \leq\right.$ and $\left.<25 \mathrm{~kg} / \mathrm{m}^{2}\right)$ & $1519(26.4)$ & $79(26.3)$ & & $1824(21.2)$ & $82(24.4)$ & \\
\hline Obesity $\left(\geq 25 \mathrm{~kg} / \mathrm{m}^{2}\right)$ & $2188(38)$ & $114(38)$ & & $2345(27.3)$ & $159(47.3)$ & \\
\hline Waist circumference, $\mathrm{cm}$ & $85.1 \pm 0.12$ & $88.2 \pm 0.51$ & $<0.001$ & $78.1 \pm 0.1$ & $85.3 \pm 0.48$ & $<0.001$ \\
\hline Fasting glucose, $\mathrm{mg} / \mathrm{dL}$ & $96.2 \pm 0.13$ & $107.2 \pm 0.81$ & $<0.001$ & $93.2 \pm 0.1$ & $106.3 \pm 0.74$ & $<0.001$ \\
\hline Glycosuria, $n$ & $33(0.6)$ & $29(9.7)$ & $<0.001$ & $20(0.2)$ & $24(7.1)$ & $<0.001$ \\
\hline Total cholesterol, mg/dL & $189.8 \pm 0.46$ & $164.8 \pm 2.03$ & $<0.001$ & $192.2 \pm 0.38$ & $177.3 \pm 2.35$ & $<0.001$ \\
\hline Triglyceride, mg/dL & $155.7 \pm 1.66$ & $147.9 \pm 5.97$ & 0.21 & $112.6 \pm 0.87$ & $143.6 \pm 4.78$ & $<0.001$ \\
\hline White blood cell, E3/ $\mu \mathrm{L}$ & $6.6 \pm 0.02$ & $6.9 \pm 0.1$ & 0.003 & $5.9 \pm 0.02$ & $6.7 \pm 0.1$ & $<0.001$ \\
\hline Hemoglobin, g/dL & $15.3 \pm 0.02$ & $14.2 \pm 0.08$ & $<0.001$ & $13.1 \pm 0.01$ & $12.8 \pm 0.07$ & $<0.001$ \\
\hline Creatinine, $\mathrm{mg} / \mathrm{dL}$ & $0.999 \pm 0.0038$ & $1.199 \pm 0.0654$ & 0.006 & $0.699 \pm 0.0023$ & $0.801 \pm 0.014$ & $<0.001$ \\
\hline \multicolumn{7}{|l|}{ 2017-18 KNHANES } \\
\hline $\mathrm{N}$ & 4015 & 181 & & 5212 & 203 & \\
\hline Age, y & $48.6 \pm 0.26$ & $66.9 \pm 0.76$ & $<0.001$ & $49.6 \pm 0.22$ & $67.8 \pm 0.72$ & $<0.001$ \\
\hline Income, $\mathbf{n}$ & & & 0.412 & & & 0.02 \\
\hline Quartile 1 & $928(23.1)$ & $51(28.2)$ & & $1233(23.7)$ & $60(29.6)$ & \\
\hline Quartile 2 & $1013(25.2)$ & $41(22.7)$ & & $1320(25.3)$ & $58(28.6)$ & \\
\hline Quartile 3 & $1032(25.7)$ & $47(26)$ & & $1289(24.7)$ & $50(24.6)$ & \\
\hline Quartile 4 & $1042(26)$ & $42(23.2)$ & & $1370(26.3)$ & $35(17.2)$ & \\
\hline Education, $\mathrm{n}$ & & & $<0.001$ & & & $<0.001$ \\
\hline Elementary & $445(11.1)$ & $63(34.8)$ & & $1066(20.5)$ & $119(58.6)$ & \\
\hline Middle school & $362(9)$ & $34(18.8)$ & & $476(9.1)$ & $29(14.3)$ & \\
\hline High school & $1381(34.4)$ & $54(29.8)$ & & $1594(30.6)$ & $39(19.2)$ & \\
\hline College & $1827(45.5)$ & $30(16.6)$ & & $2076(39.8)$ & $16(7.9)$ & \\
\hline Smoking, pack years & $12.9 \pm 0.26$ & $24.1 \pm 1.7$ & $<0.001$ & $0.6 \pm 0.06$ & $1.3 \pm 0.48$ & 0.187 \\
\hline Alcohol, g/week & $119.5 \pm 2.63$ & $89.4 \pm 11.52$ & 0.011 & $32.7 \pm 1.14$ & $12.1 \pm 4.17$ & $<0.001$ \\
\hline Sleep duration, $\mathrm{h}$ & $7.2 \pm 0.02$ & $7.4 \pm 0.11$ & 0.061 & $7.2 \pm 0.02$ & $7.2 \pm 0.11$ & 0.944 \\
\hline Hypertension, $\mathrm{n}$ & $721(18)$ & $120(66.3)$ & $<0.001$ & $866(16.6)$ & $140(69)$ & $<0.001$ \\
\hline Dyslipidemia, $\mathrm{n}$ & $310(7.7)$ & $82(45.3)$ & $<0.001$ & $573(11)$ & $102(50.2)$ & $<0.001$ \\
\hline Cardiovascular disease, $\mathrm{n}$ & $178(4.4)$ & $28(15.5)$ & $<0.001$ & $113(2.2)$ & $28(13.8)$ & $<0.001$ \\
\hline Systolic BP, mmHg & $120 \pm 0.23$ & $124.9 \pm 1.17$ & $<0.001$ & $115.7 \pm 0.24$ & $127.9 \pm 1.16$ & $<0.001$ \\
\hline Diastolic BP, mmHg & $78.2 \pm 0.16$ & $71.2 \pm 0.77$ & $<0.001$ & $73.8 \pm 0.13$ & $71.9 \pm 0.67$ & 0.006 \\
\hline Body mass index, $n$ & & & 0.081 & & & $<0.001$ \\
\hline Continued & & & & & & \\
\hline
\end{tabular}




\begin{tabular}{|l|l|l|r|l|l|r|}
\hline & \multicolumn{3}{|l|}{ Men } & \multicolumn{2}{l|}{ Women } \\
\cline { 2 - 7 } & Normal & Diabetes & P-value & Normal & Diabetes & P-value \\
\hline Normal $\left(<23 \mathrm{~kg} / \mathrm{m}^{2}\right)$ & $1342(33.4)$ & $46(25.4)$ & & $2746(52.7)$ & $57(28.1)$ & \\
\hline Pre-obesity $\left(23 \leq \mathrm{and}<25 \mathrm{~kg} / \mathrm{m}^{2}\right)$ & $1038(25.9)$ & $52(28.7)$ & & $1071(20.5)$ & $50(24.6)$ & \\
\hline Obesity $\left(\geq 25 \mathrm{~kg} / \mathrm{m}^{2}\right)$ & $1635(40.7)$ & $83(45.9)$ & & $1395(26.8)$ & $96(47.3)$ & \\
\hline Waist circumference, $\mathrm{cm}$ & $85.9 \pm 0.14$ & $88.9 \pm 0.6$ & $<0.001$ & $77.7 \pm 0.13$ & $84.9 \pm 0.6$ & $<0.001$ \\
\hline Fasting glucose, $\mathrm{mg} / \mathrm{dL}$ & $96.8 \pm 0.16$ & $109.4 \pm 0.91$ & $<0.001$ & $93.6 \pm 0.13$ & $107.1 \pm 0.95$ & $<0.001$ \\
\hline Glycosuria, $\mathrm{n}$ & $31(0.8)$ & $27(14.9)$ & $<0.001$ & $11(0.2)$ & $16(7.9)$ & $<0.001$ \\
\hline Total cholesterol, $\mathrm{mg} / \mathrm{dL}$ & $193.7 \pm 0.57$ & $155.3 \pm 2.42$ & $<0.001$ & $196 \pm 0.51$ & $165.4 \pm 2.72$ & $<0.001$ \\
\hline Triglyceride, $\mathrm{mg} / \mathrm{dL}$ & $154.9 \pm 1.89$ & $137.6 \pm 5.9$ & 0.006 & $108.9 \pm 0.99$ & $133.6 \pm 6.22$ & $<0.001$ \\
\hline White blood cell, $\mathrm{E} 3 / \mu \mathrm{L}$ & $6.5 \pm 0.03$ & $6.8 \pm 0.13$ & 0.019 & $5.8 \pm 0.02$ & $6.3 \pm 0.12$ & $<0.001$ \\
\hline Hemoglobin, $\mathrm{g} / \mathrm{dL}$ & $15.3 \pm 0.02$ & $14.4 \pm 0.11$ & $<0.001$ & $13.1 \pm 0.02$ & $12.8 \pm 0.09$ & 0.001 \\
\hline Creatinine, $\mathrm{mg} / \mathrm{dL}$ & $0.9 \pm 0$ & $1.1 \pm 0.03$ & $<0.001$ & $0.7 \pm 0$ & $0.7 \pm 0.01$ & 0.003 \\
\hline
\end{tabular}

Table 1. General characteristics of training set (2013-16 KNHANES) and testing set (2017-2018) according to gender and diabetes. Continuous and categorical variables are described as mean \pm standard error and number (percent), respectively. P-values are measured using nominal population, not weighted population. P-values of continuous and categorical variables are measured by Student t-test and Chi-squared test, respectively. KNHANES Korea National Health and Nutrition Examination Survey, BP blood pressure.

impact on predicting diabetes through machine learning techniques. High triglyceride (TG) levels showed the highest OR in women (OR 1.49; 95\% CI 1.45-1.54). High TG levels are known to be a result of metabolic dysfunction in patients with diabetes ${ }^{24}$ and a risk factor for diabetes development ${ }^{25,26}$. Recently, a rural Chinese cohort study by Yongcheng et al. reported that hypertriglyceridemia is a risk factor for diabetes ${ }^{27}$. They also suggested that reducing triglycerides can decrease the risk of developing diabetes ${ }^{27}$. This implies that a high TG level is a modifiable risk factor for diabetes and should be managed in people predisposed to diabetes.

Alcohol consumption was related to a decreased risk of diabetes in both men and women (KNHANES dataset). This finding is consistent with previous studies about alcohol consumption. Moreover, heavy and moderate consumption showed deleterious and protective effects on diabetes, respectively ${ }^{28}$. BMI and waist circumference (WC) showed positive relationships in univariate analysis. However, multivariate analysis revealed that BMI had a negative relationship, whereas WC had a positive relationship with diabetes. In light of this, waist circumference, a well-known parameter for central obesity, may be a better parameter for risk assessment of obesity than is BMI, a general obesity indicator. Wang et al. reported similar results regarding risk prediction for diabetes. According to their analysis, abdominal adiposity was superior to abdominal obesity as a predictor for new-onset diabetes ${ }^{29}$. Peter et al. also reported that WC showed higher mortality risk than BMI (WC: HR 1.40 [95\% CI 1.14-1.72] and BMI: HR 1.29 [1.04-1.61]) in adults with diabetes ${ }^{30}$.

Risk group classification is one of the most critical uses of machine learning techniques in medical research ${ }^{31}$. Using logistic regression, the combinatory effect of selected risk factors on the disease of interest could be calculated as a probability. Moreover, based on the probability obtained from LR, the participants were classified into five groups. Subsequently, we assessed the risk of each group by analyzing the cumulative incidence of diabetes using cox regression analysis. As expected, and as per our prediction model, participants at high risk showed a high incidence of diabetes (Fig. 2).

Our study had several limitations. First, we could not distinguish type 1 diabetes mellitus from type 2 diabetes mellitus because there were no biomarkers or clinical information for classifying the new-onset diabetes in the KoGES. The risk factors for each type of diabetes are different. Therefore, distinguishing the type of diabetes may be preferable when formulating a prediction model with high accuracy. However, new-onset type 1 diabetes mellitus in a patient over 30 years of age is rare ${ }^{32}$. Hence, this prediction model may be used to classify groups with a high risk for type 2 diabetes mellitus. Second, we could not use menopausal status as a predictive factor in women. The effects of various post-menopausal hormones in women must be considered ${ }^{33}$. Previous cohort studies reported controversial results regarding the role of menopausal status in diabetes development ${ }^{34,35}$. Kim et al. reported that there was no association between natural menopause and the risk for diabetes mellitus ${ }^{34}$. However, early menopause showed significant association with type 2 diabetes mellitus ${ }^{36}$. Unfortunately, KoGES data at baseline did not include the menopausal status of participants. Therefore, we could not use this factor. Third, we used two large cohort composed of Koreans. So, our diabetes index has high generalizability in Koreans, but not high in other populations. However, we had used the nationally representative surveys to establish the DM classification model. Moreover, we validated the model using the KoGES that is also a nation-wide longitudinal study. Due to setting healthy subjects as target population, our model might have the generalizability compared to other models using hospital-based participants.

In conclusion, we developed a diabetes mellitus risk classification model and validated it using Korean datasets. Although the variables used in this model cannot be counted directly, they can be easily collected in real clinical practice. Hence, this new diabetes index can be used to classify individuals at a high risk for diabetes mellitus, who should prevent the disease by managing their risks through lifestyle modification. 
Men

\begin{tabular}{|c|c|c|c|}
\hline Age, $y$ & $1.077(1.071-1.083)$ & $1.033(1.023-1.042)$ & $1.037(1.028-1.045)$ \\
\hline Income & $0.892(0.835-0.952)$ & $0.89(0.819-0.968)$ & $0.869(0.803-0.939)$ \\
\hline Education $^{\mathrm{a}}$ & $0.559(0.524-0.596)$ & $0.935(0.854-1.024)$ & NA \\
\hline Smoking, pack years & $1.293(1.244-1.345)$ & $1.041(0.996-1.087)$ & NA \\
\hline Alcohol, g/week ${ }^{\mathrm{b}}$ & $0.902(0.881-0.923)$ & $0.955(0.929-0.982)$ & $0.96(0.934-0.987)$ \\
\hline Sleep duration, $\mathrm{h}$ & $1.159(1.094-1.227)$ & $1.092(1.028-1.161)$ & $1.094(1.029-1.163)$ \\
\hline Hypertension $^{c}$ & $7.636(6.577-8.866)$ & $1.52(1.246-1.853)$ & $1.51(1.239-1.842)$ \\
\hline Dyslipidemia $^{c}$ & $8.657(7.301-10.266)$ & $2.668(2.143-3.32)$ & $2.647(2.127-3.294)$ \\
\hline Cardiovascular disease $^{\mathrm{c}}$ & $9.303(7.58-11.416)$ & $1.583(1.222-2.05)$ & $1.625(1.256-2.103)$ \\
\hline Systolic BP, mmHg ${ }^{\mathrm{b}}$ & $3.437(2.279-5.184)$ & $0.489(0.273-0.876)$ & $0.497(0.278-0.89)$ \\
\hline Diastolic BP, $\mathrm{mmHg}^{\mathrm{b}}$ & $0.064(0.045-0.092)$ & $0.526(0.309-0.894)$ & $0.512(0.301-0.87)$ \\
\hline Body mass index ${ }^{d}$ & $1.089(1-1.186)$ & $0.771(0.667-0.892)$ & $0.77(0.666-0.89)$ \\
\hline Waist circumference, $\mathrm{cm}$ & $1.044(1.036-1.052)$ & $1.051(1.037-1.065)$ & $1.051(1.037-1.065)$ \\
\hline Fasting glucose, mg/dL & $1.106(1.098-1.113)$ & $1.09(1.081-1.099)$ & $1.09(1.081-1.099)$ \\
\hline Glycosuriac $^{c}$ & $20.94(15.264-28.727)$ & $6.557(4.278-10.048)$ & $6.61(4.311-10.134)$ \\
\hline Total cholesterol, mg/dL ${ }^{b}$ & $0.06(0.047-0.078)$ & $0.144(0.106-0.196)$ & $0.143(0.105-0.194)$ \\
\hline Triglyceride, $\mathrm{mg} / \mathrm{dL}^{\mathrm{b}}$ & $0.993(0.913-1.079)$ & NA & NA \\
\hline White blood cell, E3/ $\mu \mathrm{L}^{\mathrm{b}}$ & $1.659(1.357-2.03)$ & $1.834(1.447-2.324)$ & $1.917(1.517-2.421)$ \\
\hline Hemoglobin, g/dL & $0.527(0.5-0.556)$ & $0.685(0.637-0.736)$ & $0.686(0.639-0.738)$ \\
\hline Creatinine, $\mathrm{mg} / \mathrm{dL}$ & $1.43(1.299-1.575)$ & $1.236(1.141-1.339)$ & $1.236(1.141-1.338)$ \\
\hline \multicolumn{4}{|l|}{ Women } \\
\hline Age, $y$ & $1.089(1.083-1.096)$ & $1.048(1.037-1.058)$ & $1.053(1.045-1.061)$ \\
\hline Income & $0.918(0.861-0.979)$ & $0.976(0.902-1.056)$ & NA \\
\hline Education $^{\mathrm{a}}$ & $0.417(0.389-0.446)$ & $0.924(0.833-1.026)$ & NA \\
\hline Smoking, pack years & $1.083(0.997-1.176)$ & NA & NA \\
\hline Alcohol, g/week ${ }^{\mathrm{b}}$ & $0.763(0.733-0.793)$ & $0.922(0.884-0.963)$ & $0.923(0.884-0.963)$ \\
\hline Sleep duration, $\mathrm{h}$ & $0.772(0.734-0.813)$ & $0.892(0.845-0.941)$ & $0.888(0.842-0.937)$ \\
\hline Hypertension $^{c}$ & $8.63(7.456-9.989)$ & $1.601(1.316-1.947)$ & $1.585(1.307-1.924)$ \\
\hline Dyslipidemia $^{c}$ & $7.357(6.314-8.573)$ & $1.647(1.354-2.005)$ & $1.621(1.333-1.972)$ \\
\hline Cardiovascular disease $^{c}$ & $5.511(4.272-7.11)$ & $0.912(0.668-1.244)$ & NA \\
\hline Systolic BP, mmHg ${ }^{b}$ & $18.489(13.541-25.245)$ & $1.04(0.606-1.785)$ & NA \\
\hline Diastolic BP, $\mathrm{mmHg}^{\mathrm{b}}$ & $0.514(0.358-0.739)$ & $0.385(0.238-0.623)$ & $0.399(0.263-0.605)$ \\
\hline Body mass index ${ }^{d}$ & $1.908(1.757-2.072)$ & $0.881(0.764-1.016)$ & NA \\
\hline Waist circumference, $\mathrm{cm}$ & $1.08(1.073-1.088)$ & $1.028(1.014-1.041)$ & $1.02(1.011-1.03)$ \\
\hline Fasting glucose, mg/dL & $1.122(1.115-1.13)$ & $1.093(1.085-1.102)$ & $1.094(1.085-1.102)$ \\
\hline Glycosuria $^{c}$ & $32.528(22.296-47.456)$ & $14.661(9.182-23.411)$ & $15.074(9.475-23.979)$ \\
\hline Total cholesterol, mg/dL ${ }^{\mathrm{b}}$ & $0.15(0.115-0.196)$ & $0.108(0.078-0.15)$ & $0.108(0.078-0.149)$ \\
\hline Triglyceride, $\mathrm{mg} / \mathrm{dL}^{\mathrm{b}}$ & $1.856(1.711-2.015)$ & $1.314(1.162-1.486)$ & $1.306(1.156--1.476)$ \\
\hline White blood cell, E3/ $\mu \mathrm{L}^{\mathrm{b}}$ & $3.366(2.812-4.03)$ & $2.684(2.159-3.338)$ & $2.698(2.172-3.351)$ \\
\hline Hemoglobin, g/dL & $0.809(0.767-0.854)$ & $0.734(0.686-0.784)$ & $0.73(0.684-0.779)$ \\
\hline Creatinine, $\mathrm{mg} / \mathrm{dL}$ & $1.682(1.435-1.971)$ & $1.063(0.836-1.351)$ & NA \\
\hline
\end{tabular}

Table 2. Backward stepwise logistic regression of men and women in training set. $L R$ logistic regression. ${ }^{a}$ Elementary (reference: 1)/Middle school (coded as 2)/High school (coded as 3)/College (coded as 4).

${ }^{\mathrm{b}}$ The variable is log-transformed. ${ }^{\mathrm{c}}$ Absence of status (reference: 0 )/Presence of status (coded as 1 ). ${ }^{\mathrm{d}}$ Normal (reference: 1)/Pre-obesity (coded as 2)/Obesity (coded as 3).

\section{Materials and methods}

Study population. This study used demographic data and biochemical profiles from the 2013-18 KNHANES. The KNHANES is a national surveillance system assessing the health and nutritional status of the Korean population. It is conducted annually by the Korea Centers for Disease Control and Prevention (KCDC). Details of this nationwide survey have been described elsewhere ${ }^{37}$. Subjects aged 40 years and older were included. Subjects with incomplete data regarding demographics and laboratory information were excluded. Furthermore, we excluded subjects with a fasting blood glucose level $\geq 126 \mathrm{mg} / \mathrm{dL}$ regardless of a diagnosis of diabetes mellitus. When constructing prediction models, subjects with hyperglycemia may cause bias as this may involve predicting the development of an anticipated pre-existing condition. We determined 2013-16 KNHANES data as the 


\section{(A) Korean Men}
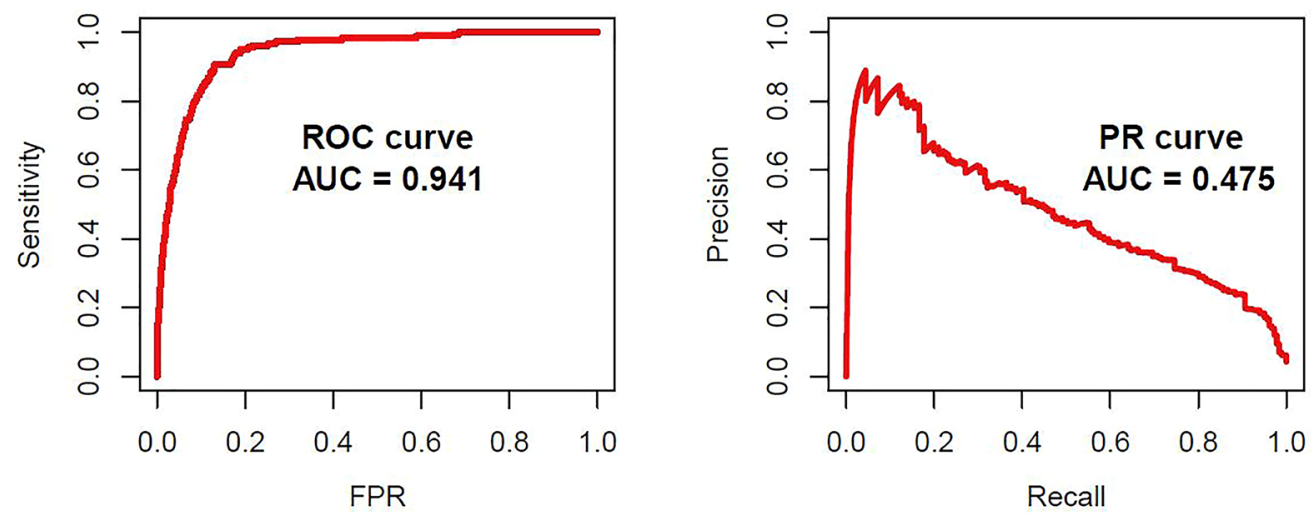

(B) Korean Women
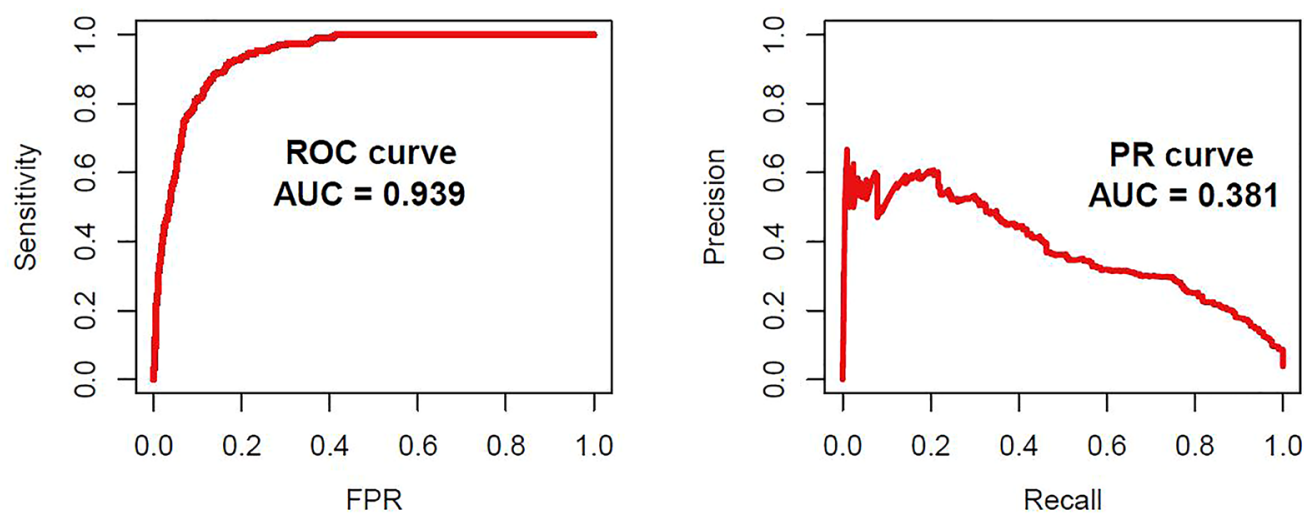

Figure 1. ROC and PR curves for the present gender-specific diabetes prediction model using the KNHANES dataset from 2017 to 2018. (A) Men (B) women. KNHANES Korea National Health and Nutrition Examination Survey, $R O C$ receiver operating characteristic, $A U C$ area under the curve, $P R$ precision recall, $F P R$ false positive rate.

derivation set and 2017-18 KNHANES data as the internal validation set. The target population of KHANES consists of nationally representative non-institutionalized civilians ${ }^{38}$.

The KoGES is an ongoing, prospective, large cohort study conducted by the Korean government. It involves a biannual examination related to life-style surveys, biochemical profiles, and incidences of common chronic diseases of Korean adults since 2001. Details of the KoGES have been described elsewhere ${ }^{39}$. We used the Ansan-Ansung cohort study, a KoGES 10-year data follow-up study, for the external validation set. Subjects who were already diagnosed with diabetes mellitus or exhibited diabetic profiles in lab tests (a fasting glucose level $\geq 126 \mathrm{mg} / \mathrm{dL}$, a 2 -h post glucose level $\geq 200 \mathrm{mg} / \mathrm{dL}$ in a $75 \mathrm{~g}$ oral glucose tolerance test [OGTT], or a glycosylated hemoglobin A1c[HbA1c] level $\geq 6.5 \%$ ) were excluded at baseline. Finally, 14,977, 9611, and 7140 subjects were used in the derivation, internal validation, and external validation sets for analysis, respectively. The major steps of inclusion/ exclusion processes of this study are described at Supplementary Fig. 1.

Definition of diabetes. Diabetes was defined according to the American Diabetes Association (ADA) guidelines ${ }^{40}$ as follows: a fasting blood glucose level $\geq 126 \mathrm{mg} / \mathrm{dL}$, a 2 -h post glucose level $\geq 200 \mathrm{mg} / \mathrm{dL}$ during OGTT, or an $\mathrm{HbAlc} \geq 6.5 \%$. Participants who were previously diagnosed as having diabetes or who exhibited diabetic features in their blood samples were categorized as the diabetes group in the KNHANES. In the KoGES, because it is a longitudinal observational study, we included non-diabetic patients in the initial cohort data. Moreover, we detected new-onset diabetes in accordance to the criteria of the ADA during the observation period.

Variable selection and statistical analysis. To determine predictive risk factors for deriving the risk prediction model, candidate variables were selected based on literature review. Two endocrinologists performed literature review and selected 40 risk factors (Supplementary Table 1). Subsequently, we determined predictive risk factors using backward stepwise logistic regression (LR) method $^{41}$ after applying weight values to all subjects 


\begin{tabular}{|c|c|c|c|c|c|c|}
\hline & \multicolumn{3}{|l|}{ Men } & \multicolumn{3}{|l|}{ Women } \\
\hline & Normal & New-onset DM & P-value & Normal & New-onset DM & P-value \\
\hline $\mathrm{N}$ & 2662 & 699 & & 3110 & 669 & \\
\hline Age, $y$ & $50.9 \pm 0.17$ & $52 \pm 0.32$ & 0.002 & $51.6 \pm 0.16$ & $53.4 \pm 0.33$ & $<0.001$ \\
\hline Income, $n$ & & & 0.697 & & & 0.142 \\
\hline Quartile 1 & $315(11.8)$ & $84(12)$ & & $693(22.3)$ & $175(26.2)$ & \\
\hline Quartile 2 & $777(29.2)$ & $208(29.8)$ & & $1025(33)$ & $216(32.3)$ & \\
\hline Quartile 3 & $962(36.1)$ & $262(37.5)$ & & $947(30.5)$ & $183(27.4)$ & \\
\hline Quartile 4 & $608(22.8)$ & $145(20.7)$ & & $445(14.3)$ & $95(14.2)$ & \\
\hline Education, $\mathbf{n}$ & & & 0.668 & & & 0.001 \\
\hline Elementary & $509(19.1)$ & $124(17.7)$ & & $1284(41.3)$ & $330(49.3)$ & \\
\hline Middle school & $597(22.4)$ & $163(23.3)$ & & $738(23.7)$ & $147(22)$ & \\
\hline High school & $964(36.2)$ & $265(37.9)$ & & $876(28.2)$ & $153(22.9)$ & \\
\hline College & $592(22.2)$ & $147(21)$ & & $212(6.8)$ & $39(5.8)$ & \\
\hline Smoking, pack years & $11.8 \pm 0.32$ & $12.8 \pm 0.64$ & 0.191 & $0.3 \pm 0.04$ & $0.4 \pm 0.12$ & 0.146 \\
\hline Alcohol, g/week & $122.5 \pm 3.74$ & $136 \pm 7.64$ & 0.112 & $9.3 \pm 0.72$ & $9.9 \pm 1.51$ & 0.716 \\
\hline Sleep duration, $\mathrm{h}$ & $6.9 \pm 0.02$ & $6.8 \pm 0.05$ & 0.189 & $6.7 \pm 0.02$ & $6.7 \pm 0.06$ & 0.881 \\
\hline Hypertension, n & $258(9.7)$ & $138(19.7)$ & $<0.001$ & $381(12.3)$ & $153(22.9)$ & $<0.001$ \\
\hline Dyslipidemia, $\mathrm{n}$ & $72(2.7)$ & $30(4.3)$ & 0.04 & $44(1.4)$ & $16(2.4)$ & 0.096 \\
\hline Cardiovascular disease, $\mathrm{n}$ & $46(1.7)$ & $12(1.7)$ & 0.999 & $31(1)$ & $18(2.7)$ & 0.001 \\
\hline Systolic BP, mmHg & $120.7 \pm 0.32$ & $125.6 \pm 0.65$ & $<0.001$ & $119.2 \pm 0.35$ & $124.7 \pm 0.76$ & $<0.001$ \\
\hline Diastolic BP, mmHg & $81.4 \pm 0.22$ & $84.3 \pm 0.42$ & $<0.001$ & $78.1 \pm 0.21$ & $81.3 \pm 0.45$ & $<0.001$ \\
\hline Body mass index, $n$ & & & $<0.001$ & & & $<0.001$ \\
\hline Normal $\left(<23 \mathrm{~kg} / \mathrm{m}^{2}\right)$ & $984(37)$ & $186(26.6)$ & & $1012(32.5)$ & $126(18.8)$ & \\
\hline Pre-obesity $\left(23 \leq\right.$ and $\left.<25 \mathrm{~kg} / \mathrm{m}^{2}\right)$ & $732(27.5)$ & $176(25.2)$ & & $835(26.8)$ & $159(23.8)$ & \\
\hline Obesity $\left(\geq 25 \mathrm{~kg} / \mathrm{m}^{2}\right)$ & $946(35.5)$ & $337(48.2)$ & & $1263(40.6)$ & $384(57.4)$ & \\
\hline Waist circumference, $\mathrm{cm}$ & $82.6 \pm 0.15$ & $85.3 \pm 0.29$ & $<0.001$ & $80.4 \pm 0.17$ & $84 \pm 0.36$ & $<0.001$ \\
\hline Fasting glucose, mg/dL & $83.3 \pm 0.16$ & $89.5 \pm 0.38$ & $<0.001$ & $80.4 \pm 0.13$ & $84.4 \pm 0.35$ & $<0.001$ \\
\hline Glycosuria, n & $107(4)$ & $76(10.9)$ & $<0.001$ & $39(1.3)$ & $25(3.7)$ & $<0.001$ \\
\hline Total cholesterol, mg/dL & $189.9 \pm 0.67$ & $195.6 \pm 1.31$ & $<0.001$ & $187.9 \pm 0.61$ & $195.1 \pm 1.27$ & $<0.001$ \\
\hline Triglyceride, mg/dL & $162.9 \pm 2.02$ & $199 \pm 5.1$ & $<0.001$ & $133.3 \pm 1.24$ & $175 \pm 3.61$ & $<0.001$ \\
\hline White blood cell, E3/ $\mu \mathrm{L}$ & $6.7 \pm 0.03$ & $6.9 \pm 0.07$ & 0.002 & $6.2 \pm 0.03$ & $6.6 \pm 0.07$ & $<0.001$ \\
\hline Hemoglobin, g/dL & $14.7 \pm 0.02$ & $14.9 \pm 0.04$ & 0.001 & $12.5 \pm 0.02$ & $12.7 \pm 0.04$ & $<0.001$ \\
\hline Creatinine, mg/dL & $0.999 \pm 0.0033$ & $1.001 \pm 0.0073$ & 0.487 & $0.7 \pm 0.0026$ & $0.7 \pm 0.0042$ & 0.971 \\
\hline
\end{tabular}

Table 3. Baseline characters of external validation set. Continuous and categorical variables are described as mean \pm standard error and number (percent), respectively. P-values are measured using nominal population, not weighted population. $\mathrm{P}$-values of continuous and categorical variables are measured by Student $\mathrm{t}$-test and Chi-squared test, respectively. $B P$ blood pressure.

in the KNHANES. Weight values were used for the processes of determining the significant risk factors and deriving the prediction model. These values were determined during data construction and denoted the subjects in the study cohort in which a number of people were represented.

Normal distribution of candidate variables was verified using the Kolmogorovo-Smirnov test. Differences in variables were analyzed based on diabetes status by means of the student's t-test and Chi-square test for continuous and categorical variables, respectively. Associations between candidate variables were analyzed separately for men and women. The LR model was used to determine the risk factors for the presence of diabetes mellitus, and to formulate the diabetes mellitus prediction model. The AUROC and the Cox regression model were used to measure the performance of the prediction model for the internal validation set and for the external validation set, respectively. Statistical analysis was performed using R language (R packages ver.3.6.1). P-value $<0.05$ was considered statistically significant.

Ethical considerations. The Institutional Review Board of Gwangju Institute of Science and Technology (South Korea) approved the study protocol (IRB No. 20200414-EX-01-02). All research procedures were performed in accordance to the relevant guidelines and regulations. All participants volunteered and provided written informed consent prior to enrolment, and their records were anonymized before being accessed by the authors. 
(A)

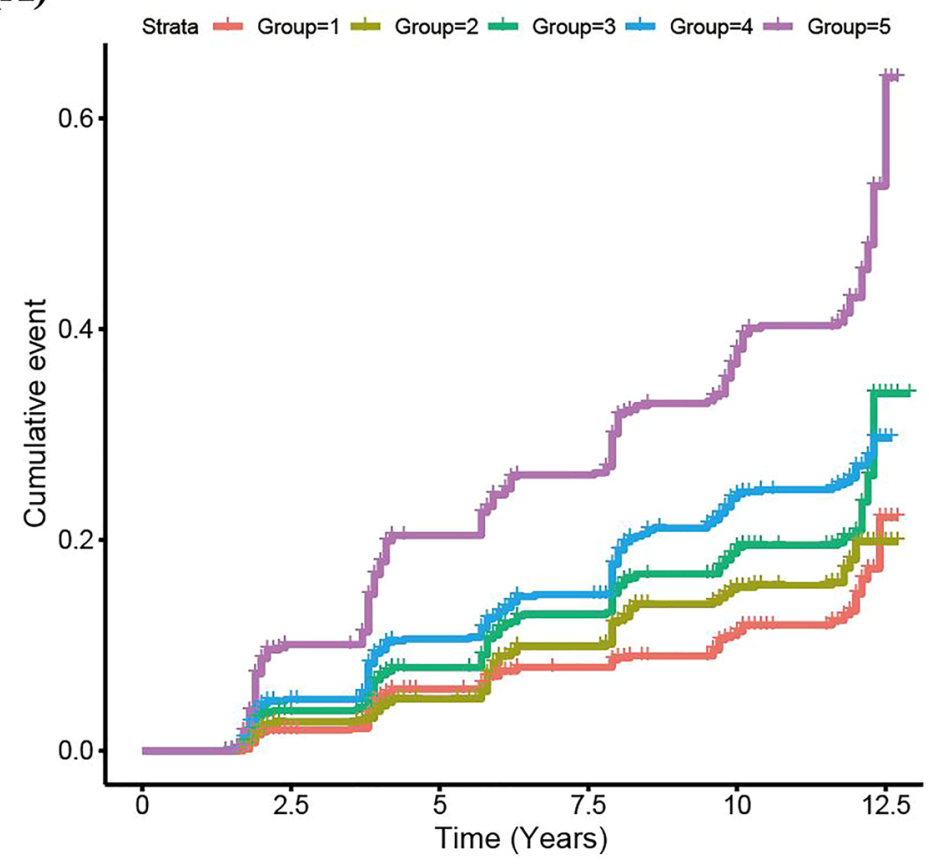

P-value matrix for comparison among five groups

\begin{tabular}{|l|c|c|c|c|}
\hline & Group 1 & Group 2 & Group 3 & Group 4 \\
\hline Group 2 & 0.918 & & & \\
\hline Group 3 & 0.003 & 0.522 & & \\
\hline Group 4 & $<0.001$ & 0.003 & 0.909 & \\
\hline Group 5 & $<0.001$ & $<0.001$ & $<0.001$ & $<0.001$ \\
\hline
\end{tabular}

(B)

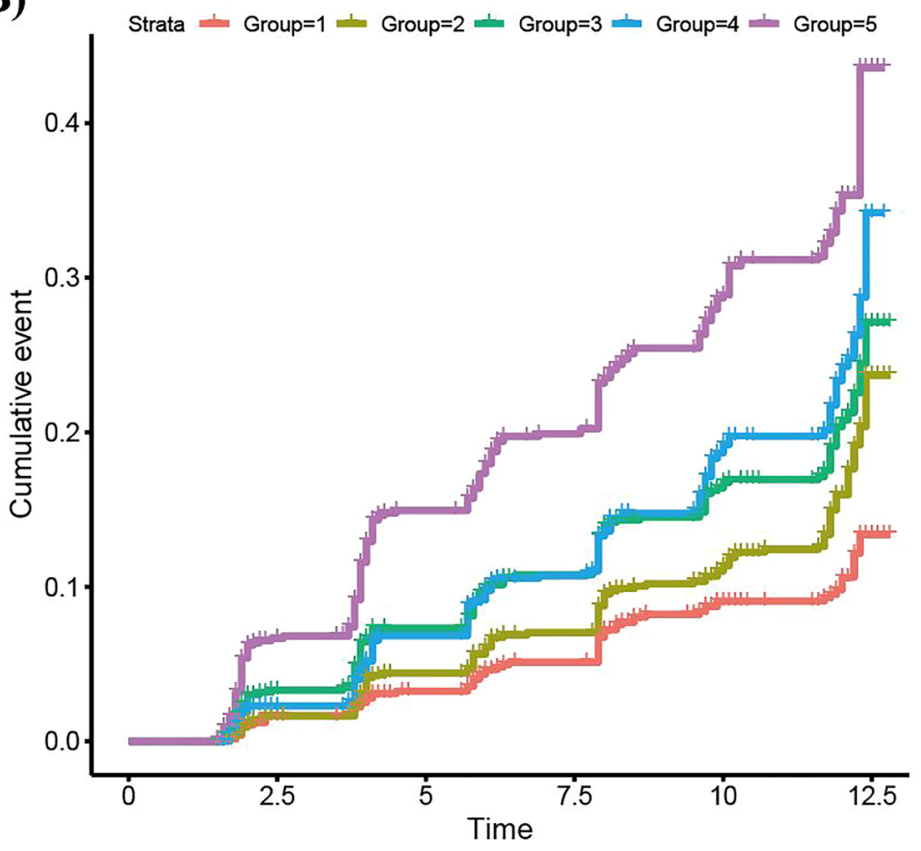

P-value matrix for comparison among five groups

\begin{tabular}{|l|c|c|c|c|}
\hline & Group 1 & Group 2 & Group 3 & Group 4 \\
\hline Group 2 & $<0.001$ & & & \\
\hline Group 3 & $<0.001$ & $<0.001$ & & \\
\hline Group 4 & $<0.001$ & $<0.001$ & 1 & \\
\hline Group 5 & $<0.001$ & $<0.001$ & $<0.001$ & $<0.001$ \\
\hline
\end{tabular}

Figure 2. Cumulative incidence difference of new-onset diabetes between five groups, divided according to the expected probabilities of participants in the KoGES study. Group 5 showed highest cumulative incidence in these five groups of both (A) men, (B) women. KoGES Korean Genome and Epidemiology Study.

Received: 28 March 2021; Accepted: 26 July 2021

Published online: 03 August 2021

\section{References}

1. Einarson, T. R., Acs, A., Ludwig, C. \& Panton, U. H. Prevalence of cardiovascular disease in type 2 diabetes: A systematic literature review of scientific evidence from across the world in 2007-2017. Cardiovasc. Diabetol. 17, 83 (2018).

2. Zheng, Y., Ley, S. H. \& Hu, F. B. Global aetiology and epidemiology of type 2 diabetes mellitus and its complications. Nat. Rev. Endocrinol. 14, 88 (2018).

3. Tuomilehto, J., Schwarz, P. \& Lindström, J. Long-term benefits from lifestyle interventions for type 2 diabetes prevention: Time to expand the efforts. Diabetes Care 34, S210-S214 (2011). 
4. Knowler, W. C. et al. Reduction in the incidence of type 2 diabetes with lifestyle intervention or metformin. N. Engl. J. Med. 346, 393-403 (2002).

5. Saito, T. et al. Lifestyle modification and prevention of type 2 diabetes in overweight Japanese with impaired fasting glucose levels: A randomized controlled trial. Arch. Intern. Med. 171, 1352-1360 (2011).

6. Pan, X.-R. et al. Effects of diet and exercise in preventing NIDDM in people with impaired glucose tolerance: The Da Qing IGT and Diabetes Study. Diabetes Care 20, 537-544 (1997).

7. Ramachandran, A. et al. The Indian Diabetes Prevention Programme shows that lifestyle modification and metformin prevent type 2 diabetes in Asian Indian subjects with impaired glucose tolerance (IDPP-1). Diabetologia 49, 289-297 (2006).

8. Carpentier, C. et al. Glycosuria amount in response to hyperglycaemia and risk for diabetic kidney disease and related events in Type 1 diabetic patients. Nephrol. Dial. Transplant. 34, 1731-1738 (2019).

9. Dhall, D., Kaur, R. \& Juneja, M. Machine learning: a review of the algorithms and its applications. Proceedings of ICRIC 2019, 47-63 (Springer, 2020).

10. Wu, H., Yang, S., Huang, Z., He, J. \& Wang, X. Type 2 diabetes mellitus prediction model based on data mining. Inform. Med. Unlocked 10, 100-107 (2018).

11. Birjais, R., Mourya, A. K., Chauhan, R. \& Kaur, H. Prediction and diagnosis of future diabetes risk: A machine learning approach. SN Appl. Sci. 1, 1112 (2019).

12. Lai, H., Huang, H., Keshavjee, K., Guergachi, A. \& Gao, X. Predictive models for diabetes mellitus using machine learning techniques. BMC Endocr. Disord. 19, 1-9 (2019).

13. Maniruzzaman, M., Rahman, M. J., Ahammed, B. \& Abedin, M. M. Classification and prediction of diabetes disease using machine learning paradigm. Health Inf. Sci. Syst. 8, 7 (2020).

14. Lim, H. M., Chia, Y. C. \& Koay, Z. L. Performance of the Finnish Diabetes Risk Score (FINDRISC) and Modified Asian FINDRISC (ModAsian FINDRISC) for screening of undiagnosed type 2 diabetes mellitus and dysglycaemia in primary care. Prim. Care Diabetes 14, 494-500 (2020).

15. Jølle, A. et al. Validity of the FINDRISC as a prediction tool for diabetes in a contemporary Norwegian population: A 10-year follow-up of the HUNT study. BMJ Open Diabetes Res. Care 7, e000769 (2019).

16. Salinero-Fort, M. et al. Performance of the Finnish Diabetes Risk Score and a simplified Finnish Diabetes Risk Score in a community-based, cross-sectional programme for screening of undiagnosed type 2 diabetes mellitus and dysglycaemia in Madrid, Spain: The SPREDIA-2 study. PLoS ONE 11, e0158489 (2016).

17. Wilson, P. W. et al. Prediction of incident diabetes mellitus in middle-aged adults: The Framingham Offspring Study. Arch. Intern. Med. 167, 1068-1074 (2007).

18. Mashayekhi, M. et al. Evaluating the performance of the Framingham Diabetes Risk Scoring Model in Canadian electronic medical records. Can. J. Diabetes 39, 152-156 (2015).

19. Zou, Q. et al. Predicting diabetes mellitus with machine learning techniques. Front. Genet. 9, 515 (2018).

20. Ha, K. H. et al. Development and validation of the Korean diabetes risk score: A 10-year national cohort study. Diabetes Metab. J. 42, 402-414 (2018).

21. Fishman, B. et al. Renal glucosuria is associated with lower body weight and lower rates of elevated systolic blood pressure: Results of a nationwide cross-sectional study of 2.5 million adolescents. Cardiovasc. Diabetol. 18, 124 (2019).

22. Ferrannini, E. Learning from glycosuria. Diabetes 60, 695-696 (2011).

23. Hung, C.-C. et al. Glycosuria and renal outcomes in patients with nondiabetic advanced chronic kidney disease. Sci. Rep. 6, 39372 (2016).

24. Mooradian, A. D. Dyslipidemia in type 2 diabetes mellitus. Nat. Rev. Endocrinol. 5, 150-159 (2009).

25. Tirosh, A. et al. Changes in triglyceride levels over time and risk of type 2 diabetes in young men. Diabetes Care 31, 2032-2037 (2008).

26. Kwon, Y. H. et al. The association between persistent hypertriglyceridemia and the risk of diabetes development: The Kangbuk Samsung Health Study. Endocrinol. Metab. 33, 55-61 (2018).

27. Ren, Y. et al. Hypertriglyceridemia-waist and risk of developing type 2 diabetes: The Rural Chinese Cohort Study. Sci. Rep. 7, 1-8 (2017).

28. Baliunas, D. O. et al. Alcohol as a risk factor for type 2 diabetes: A systematic review and meta-analysis. Diabetes Care 32, 2123-2132 (2009).

29. Wang, Y., Rimm, E. B., Stampfer, M. J., Willett, W. C. \& Hu, F. B. Comparison of abdominal adiposity and overall obesity in predicting risk of type 2 diabetes among men. Am. J. Clin. Nutr. 81, 555-563 (2005).

30. Katzmarzyk, P. T., Hu, G., Cefalu, W. T., Mire, E. \& Bouchard, C. The importance of waist circumference and BMI for mortality risk in diabetic adults. Diabetes Care 36, 3128-3130 (2013).

31. Alghamdi, M. et al. Predicting diabetes mellitus using SMOTE and ensemble machine learning approach: The Henry Ford ExercIse Testing (FIT) project. PLoS One 12, e0179805 (2017).

32. Thomas, N. J. et al. Frequency and phenotype of type 1 diabetes in the first six decades of life: A cross-sectional, genetically stratified survival analysis from UK Biobank. Lancet Diabetes Endocrinol. 6, 122-129 (2018).

33. Dalal, P. K. \& Agarwal, M. Postmenopausal syndrome. Indian J. Psychiatry 57, S222 (2015).

34. Kim, C. et al. Menopause and risk of diabetes in the Diabetes Prevention Program. Menopause (New York, NY) 18, 857 (2011).

35. Ren, Y. et al. Association of menopause and type 2 diabetes mellitus. Menopause 26, 325-330 (2019).

36. Brand, J. S. et al. Age at menopause, reproductive life span, and type 2 diabetes risk: Results from the EPIC-InterAct study. Diabetes Care 36, 1012-1019 (2013)

37. Kweon, S. et al. Data resource profile: The Korea national health and nutrition examination survey (KNHANES). Int. J. Epidemiol. 43, 69-77 (2014).

38. Kwon, S. et al. Sleep duration and mortality in Korean adults: A population-based prospective cohort study. BMC Public Health 20, $1-10(2020)$.

39. Kim, Y., Han, B.-G. \& Groiup, K. Cohort profile: The Korean genome and epidemiology study (KoGES) consortium. Int. J. Epidemiol. 46, e20-e20 (2017).

40. American Diabetes Association. 2. Classification and diagnosis of diabetes: standards of medical care in diabetes-2018. Diabetes Care 41, S13-S27 (2018).

41. Zellner, D., Keller, F. \& Zellner, G. E. Variable selection in logistic regression models. Commun. Stat. Simul. Comput. 33, 787-805 (2004).

\section{Author contributions}

S.M. and C.-M.O. developed the theory and designed the study. J.-Y.J. and Y.K. performed analysis. S.M. and C.-M.O. draft the manuscript and designed the table and figures. All authors critically reviewed the manuscript and provided critical feedback. C.-M.O. supervised the project. 


\section{Funding}

This research was supported by the Basic Science Research Program, through the National Research Foundation of Korea (2020R1C1C1004999 to C.M.O).

\section{Competing interests}

The authors declare no competing interests.

\section{Additional information}

Supplementary Information The online version contains supplementary material available at https://doi.org/ 10.1038/s41598-021-95341-8.

Correspondence and requests for materials should be addressed to C.-M.O.

Reprints and permissions information is available at www.nature.com/reprints.

Publisher's note Springer Nature remains neutral with regard to jurisdictional claims in published maps and institutional affiliations.

(c) (i) Open Access This article is licensed under a Creative Commons Attribution 4.0 International License, which permits use, sharing, adaptation, distribution and reproduction in any medium or format, as long as you give appropriate credit to the original author(s) and the source, provide a link to the Creative Commons licence, and indicate if changes were made. The images or other third party material in this article are included in the article's Creative Commons licence, unless indicated otherwise in a credit line to the material. If material is not included in the article's Creative Commons licence and your intended use is not permitted by statutory regulation or exceeds the permitted use, you will need to obtain permission directly from the copyright holder. To view a copy of this licence, visit http://creativecommons.org/licenses/by/4.0/.

(C) The Author(s) 2021 